1

\title{
Detection of an unstable minor QTL linked to fire blight resistance on linkage group 16 of Malus fusca
}

\section{Ofere Francis Emeriewen ${ }^{1 *}$, Klaus Richter ${ }^{2}$, Annette Wensing ${ }^{3}$, Mickael Malnoy ${ }^{4}$ and Andreas Peil ${ }^{1 *}$}

${ }^{1}$ Julius Kühn Institute (JKI), Federal Research Centre for Cultivated Plants, Institute for Breeding Research on Fruit Crops, Dresden, Germany

${ }^{2}$ Julius Kühn Institute (JKI), Federal Research Centre for Cultivated Plants, Institute for Resistance Research and Stress Tolerance, Quedlinburg, Germany

${ }^{3}$ Julius Kühn Institute (JKI), Federal Research Centre for Cultivated Plants, Institute for Plant Protection in Fruit Crops and Viticulture, Dossenheim, Germany

${ }^{4}$ Research and Innovation Centre, Fondazione Edmund Mach (FEM), San Michele all 'Adige, Italy

*Correspondence: ofere.emeriewen@julius-kuehn.de; andreas.peil@julius-kuehn.de

\begin{abstract}
Objective

Erwinia amylovora causes fire blight disease in Malus. A strong resistance QTL (Mfu10) was previously detected on linkage group 10 of Malus fusca accession MAL0045, using several strains of the bacterium. As no strain capable of breaking the resistance of MAL0045 has been found, it was hypothesized that a second resistance factor contributes to the fire blight resistance of MAL0045. However, to date, no minor locus has been detected with previously published strains of the bacterium. We detected a minor QTL only on a subset of a population following inoculation with strain Ea1038, which heterologously expresses an effector in a derivative of isolate Ea3049. Two genetic maps of MAL0045, one scarce, the other dense with markers, were used for QTL analyses.
\end{abstract}

\section{Results}

Mful0 was detected on LG10 with Ea1038, as was previously with Ea3049. Although no other QTLs of significant LOD was previously detected in other linkage groups with Ea3049, a QTL of significant LOD was detected on LG16 (Mful6) after inoculation of a subset of 76 individuals with Ea1038, but only using the dense genetic map. Mful6 improved the effect of Mfu10. However, when the number of individuals inoculated with Ea1038 was increased to 121, Mful6 was no longer detected in the dense genetic map. We hypothesize some factors, which might be responsible for the instability of this QTL.

Keywords: Erwinia amylovora strains, MAL0045, Mfu10, Mfu16, Malus fusca fire blight resistance loci 


\section{Introduction}

The bacterium, Erwinia amylovora, causes fire blight - a devastating disease of the domesticated apple (Malus domestica Borkh.) and related species (Malus spp.) [1-4]. The mechanisms by which the pathogen invades and causes disease in susceptible hosts have been extensively reviewed $[2,5]$. Similarly, the molecular strategies employed by resistant hosts for the recognition of E. amylovora elicitors are well documented [2, 4, 6]. Malus host resistance is mostly quantitative, evidenced by the segregation of resistant and susceptible phenotypes in Malus populations [7-11]. Quantitative trait loci (QTLs) for fire blight resistance have been detected in apple cultivars and wild apple species accessions [reviewed in 4]. Although most apple cultivars are more susceptible to the disease, their QTLs are faster to introgress but not sufficient to provide strong resistance as those of their wild relatives [8-11], for which fire blight resistance candidate genes have been proposed [12-15], and in one instance, functionally proven [16].

A strong fire blight resistance QTL (Mfu10) was detected on linkage group (LG) 10 of the wild apple accession, Malus fusca MAL0045 [9]. The stability of MfulO has been demonstrated using several strains of $E$. amylovora differing in virulence/aggressiveness [17-19]. In particular, the highly aggressive Canadian strain, Ea3049, and the mutant strain ZYRKD3-1, both of which break down the resistance locus of another wild Malus genotype, Malus $\times$ robusta 5 (Mr5) [20, 21], could not breakdown Mfu10 [17, 18]. However, unlike in Mr5, where minor QTLs were detected following inoculation with Ea3049 [22], no minor QTLs were detected in MAL0045 following inoculations with four different strains [19].

\section{Rationale and methods}

It was previously reported that a switch from cysteine amino acid (C-allele) to serine amino acid (S-allele) in the avrRpt2 $2_{\mathrm{EA}}$ effector protein sequence of E. amylovora at position 156 is responsible for virulence and resistance breakdown in Mr5 [21] but also aggressiveness in other Malus host [4]. Ea3049 possesses the S-allele contributing to the high virulence/aggressiveness of this strain.

That MAL0045 itself is highly resistant to Ea3049 but the average PLL of the progeny increased to 62.4 compared to of 22.6 after inoculation with strain Ea222 [17], led us to speculate that there might be a second factor contributing to the resistance of MAL0045, and/or the SNP in the avrRpt2 $2_{\mathrm{EA}}$ effector of Ea3049 [21] might not be the only virulence factor of this strain. We inoculated the original mapping population (05210 individuals) derived from MAL0045 $\times$ 'Idared' cross [9], with a mutant strain of Ea3049. In the meantime, we developed a dense genetic map of MAL0045 using genotyping-by-sequencing generated SNPs incorporated with microsatellite markers (SSRs), for MAL0045-derived progenies namely: 05210 and 09260 individuals [19].

The strain used in this study, Ea1038, is a derivative of Ea3049 with the chromosomal S-allele of the avrRpt $2 \mathrm{EA}$ effector deleted and complemented with the $\mathrm{C}$-allele on an expression vector. Artificial shoot inoculation was performed on scions of up to 10 replicates of each progeny individual grafted onto rootstock M9, by cutting the youngest leaves with a pair of scissors dipped into bacterial inoculum $\left(10^{9} \mathrm{cfu} / \mathrm{ml}\right)$. Disease necrosis was measured in centimeters 27 
days post inoculation (dpi) and converted into percent lesion length (PLL) by dividing the necrotic shoot by the total shoot length and multiplying by 100. The preliminary QTL mapping was done with the phenotypic data of 76 inoculated plants. Subsequently, the number of phenotyped individuals could be increased to 121 individuals. These data were used for a second QTL mapping. The calculated average of PLL of all replicates of each individual was used for Kruskal-Wallis analysis and interval mapping using MapQTL 5.0 [23]. The first incomplete genetic map of MAL0045 [9, 24] and the recently developed dense map [19] served as templates for QTL analysis. This map was established using 112 individuals of the 05210 population and 36 individuals of the 09260 population, 148 individuals in total.

SAS (SAS Institute) GLIMMIX (generalized linear mixed model) analysis was performed to determine whether the effects of detected loci were significantly different. For this analysis, phenotypic values (PLL) of each progeny individual as well as their marker alleles were employed.

\section{Results}

Of 112 individuals of 05210 population used to develop the map [19], it was only possible to phenotype 76 individuals by artificial shoot inoculation with Ea1038 in 2017. The distribution of PLLs for all 76 individuals is shown in Figure 1. For these individuals, $61.1 \%$ and $65.7 \%$ were the mean and median PLLs, respectively. Only five individuals recorded PLLs below 10.0 with 3.3 the lowest. Fifty-two individuals recorded PLLs over 50 with 100 recorded as the highest for twelve individuals. For the parents, whilst a PLL of 9.6 was recorded for MAL0045, $100 \%$ was recorded for 'Idared'.

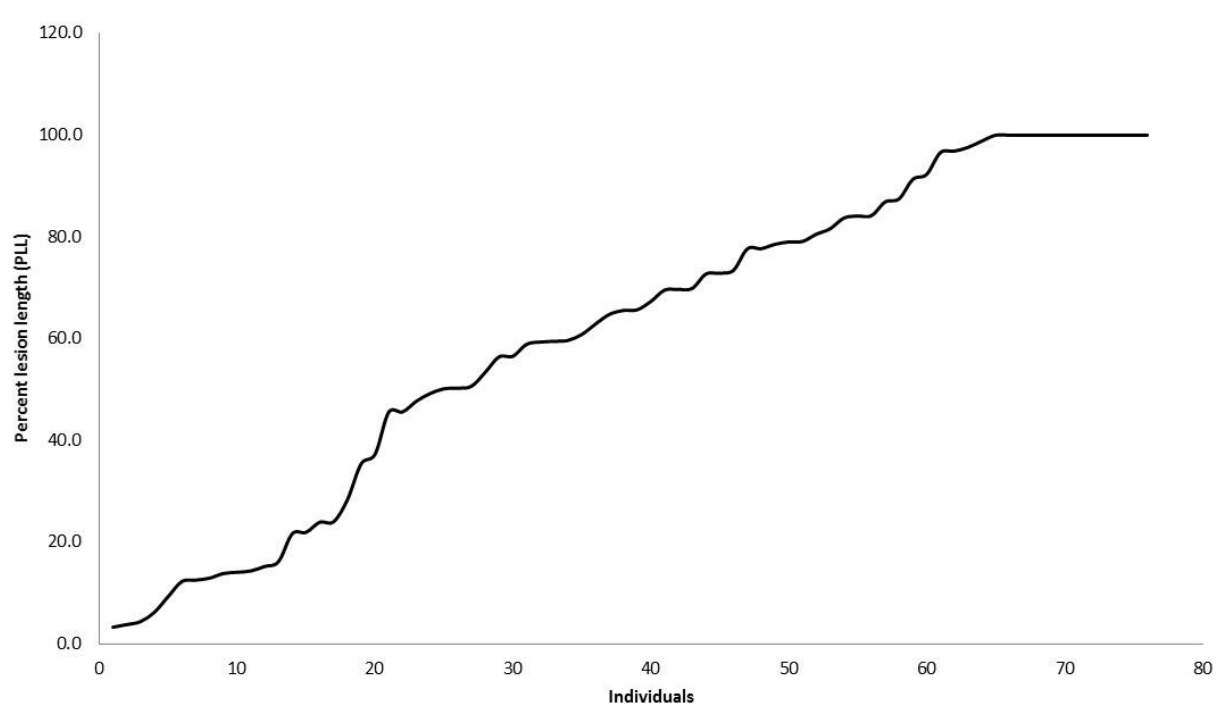

Figure 1. Distribution of PLL of seventy-six individuals of 05210 population phenotyped with Ea1038 
The averages of all replicates for each of the 76 individuals were used for Kruskal-Wallis analysis and interval mapping with the newly developed map as template. Kruskal-Wallis analysis showed that markers on LG10 (highest $K$ value $=37.2$ ), LG16 (highest $K=13.0$ ) and LG17 (highest $K=11.6$ ) correlated with fire blight resistance (Table 1). The most significant correlation with resistance was observed in LG10 where individuals inheriting the resistant allele of the marker with the highest $K$ value (Sca_304010_602250) possessed $43.8 \%$ less necrosis than individuals inheriting the susceptible allele (i.e. difference between mean 11 and mean $1 \mathrm{~m}$ genotypic segregation). For LG16 and LG17, the differences were $28.1 \%$ (Sca_304000_613165) and 22.1\% (CH05b06_L4), respectively (Table 1). With the first incomplete map, only markers on LG10 showed correlation with fire blight resistance (data not shown).

Table 1. Kruskal-Wallis analysis results for some markers on the three linkage groups of the dense genetic map showing strong correlation with fire blight resistance following inoculation with Ea1038

\begin{tabular}{|c|c|c|c|c|c|}
\hline \multirow[t]{2}{*}{ Marker } & \multirow[t]{2}{*}{ LG } & \multirow{2}{*}{$\begin{array}{l}\text { Position } \\
\text { (cM) }\end{array}$} & \multirow[t]{2}{*}{$K$} & \multicolumn{2}{|l|}{ PLLs of plants with } \\
\hline & & & & Susceptible alleles & Resistance alleles \\
\hline CH03d11 & LG10 & 38.127 & $35.6 * * * * * * *$ & 85.1 & 42.2 \\
\hline Sca_313304_278642 & LG10 & 39.404 & $36.2 * * * * * * *$ & 84.9 & 41.8 \\
\hline FR481A & LG10 & 41.421 & $31.3 * * * * * * *$ & 84.2 & 44.4 \\
\hline Sca_304010_602250 & LG10 & 43.152 & $37.2 * * * * * * *$ & 84.7 & 40.9 \\
\hline FR149B & LG10 & 48.740 & $27.8 * * * * * * *$ & 81.7 & 44.7 \\
\hline Sca_300922_5406043 & LG16 & 12.568 & $11.6 * * * * *$ & 76.8 & 50.3 \\
\hline Sca_315074_14818 & LG16 & 14.715 & $12.9 * * * * * *$ & 76.8 & 49.1 \\
\hline Sca_304000_613165 & LG16 & 16.650 & $13.0 * * * * * *$ & 76.8 & 48.7 \\
\hline Sca_300922_6119061 & LG16 & 16.013 & $12.8 * * * * * *$ & 76.5 & 48.7 \\
\hline Sca_315325_42556 & LG17 & 0.000 & $11.2 * * * * *$ & 72.8 & 50.1 \\
\hline Sca_313414_17371 & LG17 & 2.172 & $10.9 * * * * *$ & 73.2 & 51.2 \\
\hline Sca_307499_834166 & LG17 & 2.204 & $10.9 * * * * *$ & 72.9 & 51.2 \\
\hline CH05b06_L4 & LG17 & 2.808 & $11.6 * * * * *$ & 72.1 & 50.0 \\
\hline
\end{tabular}


Interval mapping with the genome wide (GW) threshold of 4.9 identified two QTLs of significant LOD scores on LG10 and LG16. No significant QTL was found on LG17. The QTL detected on LG10 is Mful0, previously detected with other strains of E. amylovora since it is located in the same interval between CH03d11 and FR149B with markers possessing highly significant $K$ values $(P=0.0001)$ (Table 1). However, the QTL on LG16 (Mful6) is a novel minor QTL (Figure 2) never previously detected with any strain. Mful6 was not detected in the scarce map with Ea1038. Interval mapping results was in agreement with Kruskal-Wallis analysis as SNP markers on LG16 with highly significant $K$ values (Table 1) possessed the highest LODs and appear underneath the QTL plot (Figure 2). The significance and interaction between $M f u 10$ and Mful6 were determined using SAS GLIMMIX analysis. Fire blight resistance was significantly stronger when resistance alleles of both loci were present in individuals compared to when individuals possessed only $M f u 10$ or Mful6 resistance alleles (Figure 3). Further, the resistance level of $M f u 10$ alone was significantly stronger than the resistance level of Mful6 alone.

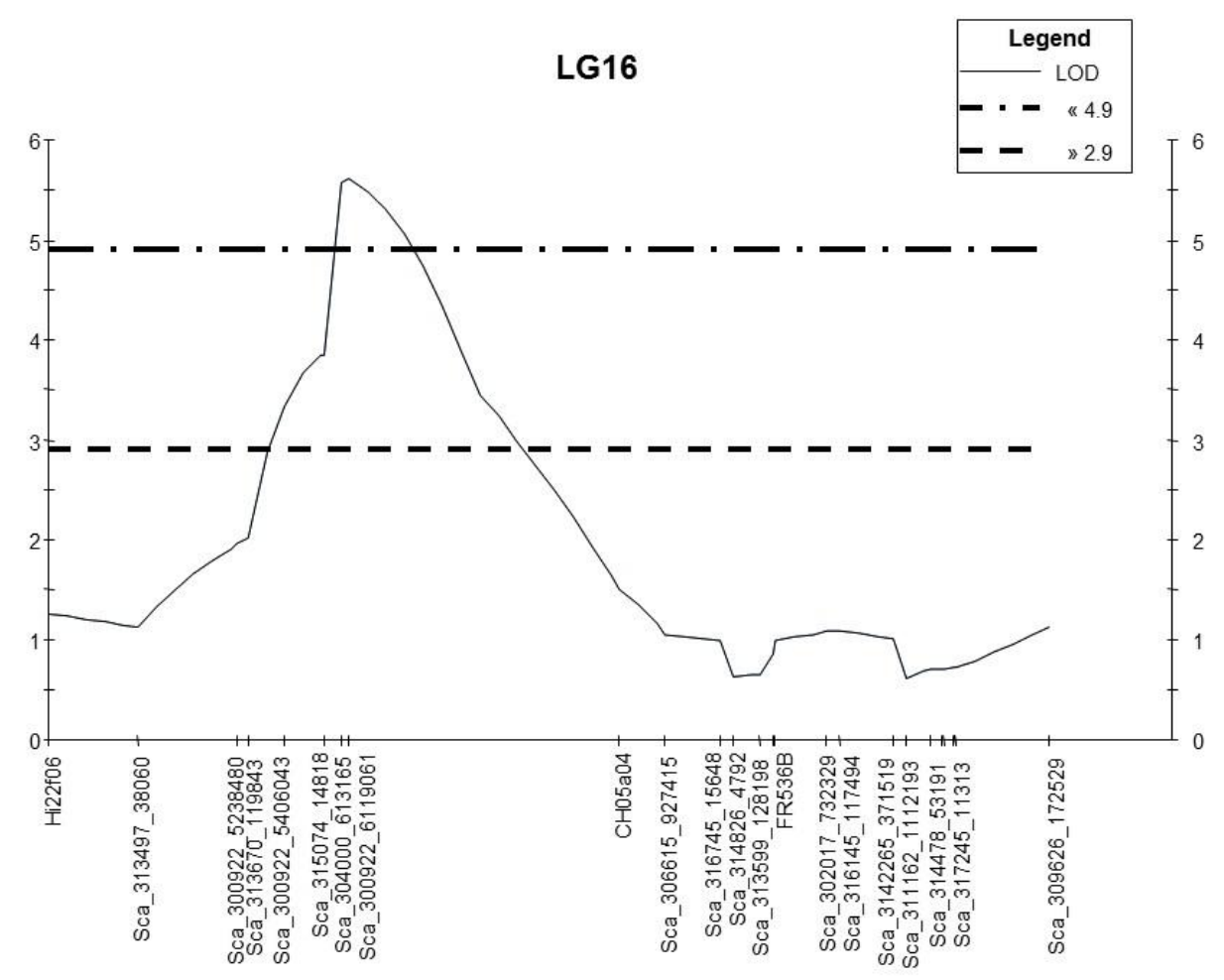

Figure 2. LOD plot of interval mapping for the detected QTL on LG16 showing the significance at the 
147 A total of 121 individuals could be inoculated with Ea1038 from 2017 to 2020. The mean PLL 148 calculated for the 121 individuals was 55.30 with a median of 59.38. Nine individuals recorded 149 PLLs below 10.0 with 1.09 being the lowest in only one individual. Seventy individuals recorded PLLs over 50 with 100 recorded as the highest for five individuals. QTL analysis with these data resulted in the detection of MfulO (data not shown) but not Mful6.

LGs

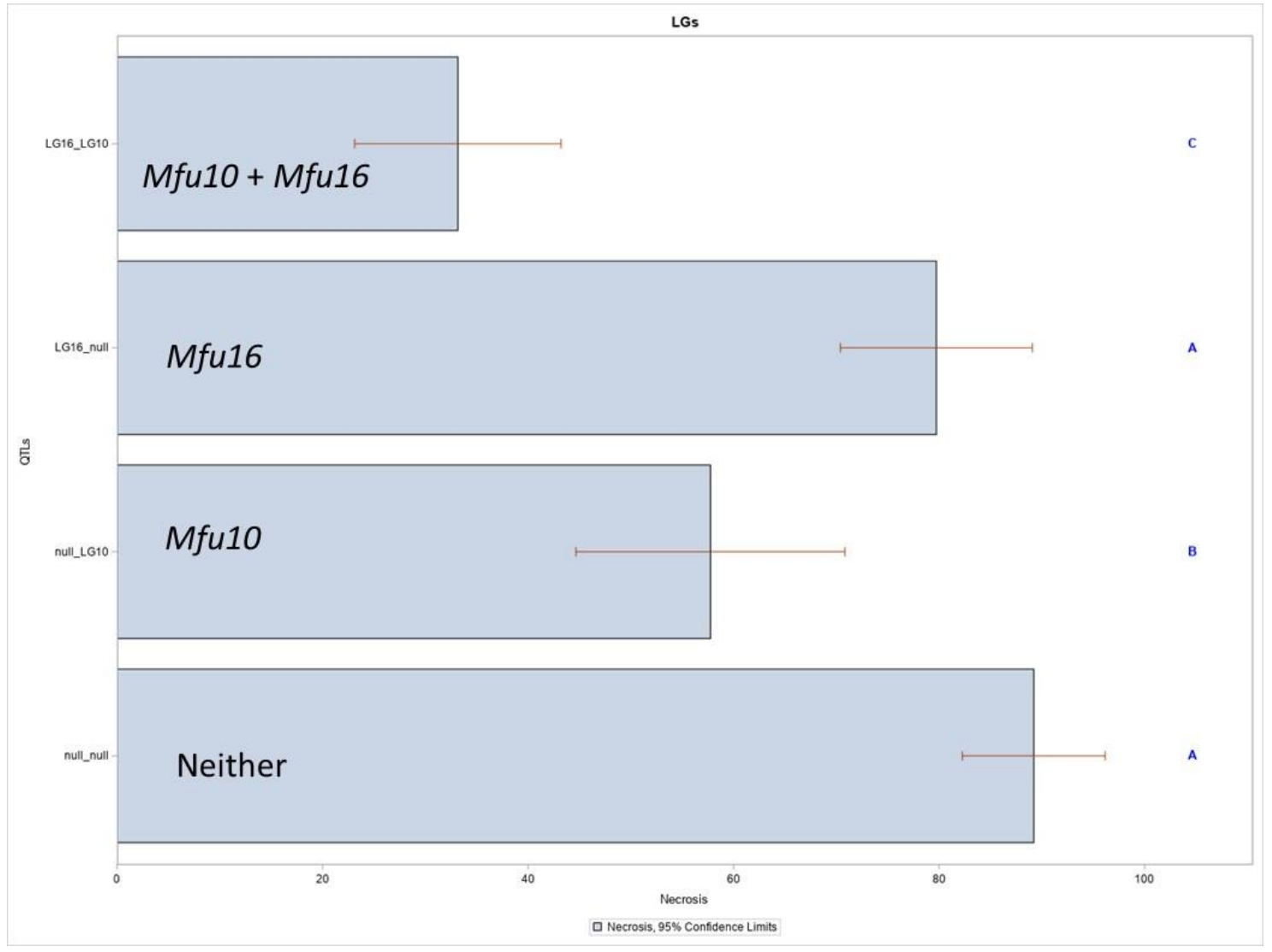

Figure 3. Significance and interaction between $M f u 10$ and $M f u 16$ determined by SAS GLIMMIX analysis 


\section{Discussion}

158

159

160

161

162

163

164

165

166

167

168

169

170

171

172

173

174

175

176

177

178

179

180

181

182

183

184

185

186

187

188

189

190

191

192

193

194

195

196

197

198

We study the interaction of M. fusca (MAL0045) and derived progeny with different strains of E. amylovora through artificial inoculation and QTL mapping. Through this process, MfulO was first identified on LG10 using Ea222 [9, 24]. The highly virulent Canadian strain, Ea3049, affected $M f u 10$ but did not overcome it, although this strain was not aggressive on MAL0045 $[17,19]$. We therefore hypothesized a second putative resistance factor, possibly another locus, might be involved in the resistance of MAL0045. The failure to detect another locus was partly attributed to the fact that the first genetic map of MAL0045 was only scarce and did not represent the whole genome $[9,24]$. This hypothesis was predicated on the situation in Mr5 where, although one minor QTL was detected on LG5 after inoculation with Ea3049 [20], a few more minor QTLs were detected following the development of a more saturated genetic map and inoculation with different strains [22]. The first map of MAL0045 [9, 24], developed with only the 05210 population, consists of 213 loci made up of DArT markers, a few SNPs and SSRs developed from the apple genome [25]. On the other hand, using tunable genotypingby-sequencing technology (tGBS), thousands of de novo SNP markers were developed for MAL0045, of which 560 SNPs were mapped including 53 SSR markers [19]. Thus, the dense map has 400 markers more than the initial map and correctly represents the genome of MAL0045. However, no minor fire blight locus were detected with this map [19]. Since fire blight resistance is strain-dependent [21], we therefore speculated that the failure to detect a minor locus is not only dependent on the marker density of the genetic map, but also the interaction with the given E. amylovora strain used for inoculation.

Ea1038 phenotypic results showed that this mutant strain was as virulent as the wild type, Ea3049 [17] with more than half of the 76 individuals having PLLs above $50 \%$. The mean PLL of 61.7 with Ea1038 is similar to 62.4 recorded with Ea3049 on the 05210 population. The results are also similar to the effect of Ea3049 on another wild apple M. ×arnoldiana MAL0004 [11], where the mean PLL recorded for 87 individuals was $65.9 \%$. It is interesting to note that although this mutant is complemented with the $\mathrm{C}$-allele, it was still very aggressive to the individuals inoculated. This suggests that the switch from cysteine amino acid (C-allele) to serine amino acid (S-allele) at position 156 of avrRpt2 EA amino acid sequence [21] is not the only factor that contributes to the pathogenicity of S-allele strains. Nevertheless, we detected two QTLs of significant LODs on two different linkage groups, LG10 and LG16. It was quite clear that MfulO is QTL located on LG10, however, a novel minor fire blight QTL, never previously detected with any strain was located on LG16 (Mful6). We propose Mful6 as a minor fire blight locus albeit unstable. Although, Mful6 was detected only with the subset of 76 individuals, and independently did not contribute significantly to resistance levels, it positively affects $M f u 10$, as the effect of both loci is significantly stronger than $M f u 10$ alone in the 05210 individuals. Minor fire blight QTLs were detected on LGs 5, 7, 11, and 14 of Mr5 [22], however, only the minor QTL on LG7 was found to contribute to resistance in addition to the major QTL on LG3 [10, 26].

Both the strain and the dense map were important factors in detecting Mful6 in this study. That this mutant strain and not the wild type, Ea3049, led to the detection of Mful6 is indicative of strong incompatible interaction between $M$. fusca and the C-allele of the avrRpt2EA effector of 
199 E. amylovora. In addition, the failure to detect Mful6 in the initially developed map [9] is

200 indicative of the important role dense genetic maps play in molecular genetics studies in Malus

201 species and other plant species.

\section{Limitations}

203 The failure to detect Mful6 following the addition of more phenotypic data is somewhat of a

204 surprise and frankly unexplainable result, although we do not rule out the role of individuals in

205 the map with missing phenotypic data. Some of the individuals with missing phenotypic data

206 died off in the orchard and hence could not be phenotyped. It cannot be excluded that the

207 exchange of genotypes during scion cutting, grafting, inoculation or measuring could be a

208 reason, too. Nevertheless, the results are strong indications of a putative minor QTL on LG16

209 of MAL0045, which significantly improves the resistance of Mfu10. In the following years,

210 more phenotypic evaluation of these individuals and other established crosses with MAL0045-

211 derived progeny will help determine the usefulness and stability of Mful6. 


\section{Supplementary Information}

Not applicable.

\section{Abbreviations}

cfu/ml: colony forming units/milliliter; dpi: days post inoculations; GW: genome wide; GLIMMIX: generalized linear mixed model; LG: linkage group; LOD: logarithm of the odds; PLL: percent lesion length; QTL: quantitative trait locus; SNP: single nucleotide polymorphisms; SSRs: simple sequence repeats;

\section{Ethics approval and consent to participate}

Not applicable

\section{Availability of data and material}

Data generated from this study are published within this article. Further materials can be provided on request from the corresponding authors, Ofere Francis Emeriewen and Andreas Peil.

\section{Funding}

232 Ofere Francis Emeriewen is funded by DFG (Deutsche Forschungsgemeinschaft) research grant received - project number AOBJ 661177.

\section{Acknowledgements}

236 We acknowledge DFG for providing funding. We thank the orchard staff at Dresden-Pillnitz and Quedlinburg for excellent technical assistance.

\section{Consent for publication}

We confirm that all authors read and approved this manuscript for publication

\section{Competing interests}

243 The authors' declare no competing interests

\section{Authors' contributions}

247 AP, OFE and MM for concept of the research, AP established the populations, OFE is responsible for the project and AP supervises the research; AW developed the mutant strain,

249 KR performed the inoculations, AP and OFE performed mapping analyses, OFE and AP prepared the manuscript, and all authors read and approved the manuscript. 


\section{References}

1. Norelli JL, Jones AL, Aldwinckle HS. Fire blight management in the twenty-first century: using new technologies that enhance host resistance in apple. Plant Disease. 2003 Jul;87(7):756-65. https://doi.org/10.1094/PDIS.2003.87.7.756

2. Malnoy M, Martens S, Norelli JL, Barny MA, Sundin GW, Smits TH, Duffy B. Fire blight: applied genomic insights of the pathogen and host. Annual review of phytopathology. 2012 Sep 8;50:475-94. https://doi.org/10.1146/annurev-phyto$\underline{081211-172931}$

3. Peil A, Emeriewen OF, Khan A, Kostick S, Malnoy M. Status of fire blight resistance breeding in Malus. Journal of Plant Pathology. 2020 Jun 5:1-0. https://doi.org/10.1007/s42161-020-00581-8

4. Emeriewen OF, Wöhner T, Flachowsky H, Peil A. Malus hosts-Erwinia amylovora interactions: strain pathogenicity and resistance mechanisms. Frontiers in plant science. 2019 Apr 26;10:551. https://doi.org/10.3389/fpls.2019.00551

5. Yuan X, Hulin MT, Sundin GW. Effectors, chaperones, and harpins of the Type III secretion system in the fire blight pathogen Erwinia amylovora: a review. Journal of Plant Pathology. 2020 Aug 11:1-5. https://doi.org/10.1007/s42161-020-00623-1

6. Khan MA, Zhao YF, Korban SS. Molecular mechanisms of pathogenesis and resistance to the bacterial pathogen Erwinia amylovora, causal agent of fire blight disease in Rosaceae. Plant Molecular Biology Reporter. 2012 Apr 1;30(2):247-60. DOI $\underline{10.1007 / \mathrm{s} 11105-011-0334-1}$

7. Calenge F, Drouet D, Denancé C, Van de Weg WE, Brisset MN, Paulin JP, Durel CE. Identification of a major QTL together with several minor additive or epistatic QTLs for resistance to fire blight in apple in two related progenies. Theoretical and Applied Genetics. 2005 Jun 1;111(1):128-35. DOI 10.1007/s00122-005-2002-Z

8. Durel CE, Denance C, Brisset MN. Two distinct major QTL for resistance to fire blight co-localize on linkage group 12 in apple genotypes 'Evereste' and Malus floribunda clone 821. Genome. 2009 Feb;52(2):139-47. https://doi.org/10.1139/G08-111

9. Emeriewen O, Richter K, Kilian A, Zini E, Hanke MV, Malnoy M, Peil A. Identification of a major quantitative trait locus for resistance to fire blight in the wild apple species Malus fusca. Molecular Breeding. 2014 Aug;34(2):407-19. DOI 10.1007/s11032-014$\underline{\text { 0043-1 }}$

10. Peil A, Garcia-Libreros T, Richter K, Trognitz FC, Trognitz B, Hanke MV, Flachowsky $\mathrm{H}$. Strong evidence for a fire blight resistance gene of Malus robusta located on linkage group 3. Plant Breeding. 2007 Oct;126(5):470-5. doi:10.1111/j.1439$\underline{0523.2007 .01408 . x}$

11. Emeriewen OF, Peil A, Richter K, Zini E, Hanke MV, Malnoy M. Fire blight resistance of Malus xarnoldiana is controlled by a quantitative trait locus located at the distal end of linkage group 12. European Journal of Plant Pathology. 2017 Aug;148(4):1011-8. DOI 10.1007/s10658-017-1152-6

12. Parravicini G, Gessler C, Denance C, Lasserre-Zuber PA, Vergne E, Brisset MN, Patocchi A, Durel CE, Broggini GA. Identification of serine/threonine kinase and nucleotide-binding site-leucine-rich repeat (NBS-LRR) genes in the fire blight 
resistance quantitative trait locus of apple cultivar 'Evereste'. Molecular plant pathology. 2011 Jun;12(5):493-505. DOI: 10.1111/J.1364-3703.2010.00690.X

13. Fahrentrapp J, Broggini GA, Kellerhals M, Peil A, Richter K, Zini E, Gessler C. A candidate gene for fire blight resistance in Malus $\times$ robusta 5 is coding for a CC-NBSLRR. Tree genetics \& genomes. 2013 Feb;9(1):237-51. DOI 10.1007/s11295-0120550-3

14. Emeriewen OF, Richter K, Piazza S, Micheletti D, Broggini GA, Berner T, Keilwagen J, Hanke MV, Malnoy M, Peil A. Towards map-based cloning of FB_MfulO: identification of a receptor-like kinase candidate gene underlying the Malus fusca fire blight resistance locus on linkage group 10. Molecular breeding. 2018 Aug;38(8):1-4. https://doi.org/10.1007/s11032-018-0863-5

15. Emeriewen OF, Richter K, Flachowsky H, Malnoy M, Peil A. Genetic analysis and fine mapping of the fire blight resistance locus of Malus xarnoldiana on linkage group 12 reveal first candidate genes. Frontiers in Plant Science 2021 April https://doi.org/10.3389/fpls.2021.667133

16. Broggini GA, Wöhner T, Fahrentrapp J, Kost TD, Flachowsky H, Peil A, Hanke MV, Richter K, Patocchi A, Gessler C. Engineering fire blight resistance into the apple cultivar 'Gala'using the FB _ MR 5 CC-NBS-LRR resistance gene of Malus $\times$ robusta 5. Plant biotechnology journal. 2014 Aug;12(6):728-33. doi: 10.1111/pbi.12177

17. Emeriewen OF, Richter K, Hanke MV, Malnoy M, Peil A. The fire blight resistance QTL of Malus fusca (Mfu10) is affected but not broken down by the highly virulent Canadian Erwinia amylovora strain E2002A. European journal of plant pathology. 2015 Mar;141(3):631-5. DOI 10.1007/s10658-014-0565-8

18. Emeriewen OF, Richter K, Hanke MV, Malnoy M, Peil A. Further insights into Malus fusca fire blight resistance. Journal of Plant Pathology. 2017 Oct 1:45-9. https://www.jstor.org/stable/45156718

19. Emeriewen OF, Richter K, Berner T, Keilwagen J, Schnable PS, Malnoy M, Peil A. Construction of a dense genetic map of the Malus fusca fire blight resistant accession MAL0045 using tunable genotyping-by-sequencing SNPs and microsatellites. Scientific reports. 2020 Oct 1;10(1):1-0. https://doi.org/10.1038/s41598-020-73393-6

20. Peil A, Flachowsky H, Hanke MV, Richter K, Rode J. Inoculation of Malus $\times$ robusta 5 progeny with a strain breaking resistance to fire blight reveals a minor QTL on LG5. InXII International Workshop on Fire Blight 8962010 Aug 16 (pp. 357-362).

21. Vogt I, Wöhner T, Richter K, Flachowsky H, Sundin GW, Wensing A, Savory EA, Geider K, Day B, Hanke MV, Peil A. Gene-for-gene relationship in the host-pathogen system Malus xrobusta 5-Erwinia amylovora. New Phytologist. 2013 Mar;197(4):1262-75. doi: 10.1111/nph.12094

22. Wöhner TW, Flachowsky H, Richter K, Garcia-Libreros T, Trognitz F, Hanke MV, Peil A. QTL mapping of fire blight resistance in Malus $\times$ robusta 5 after inoculation with different strains of Erwinia amylovora. Molecular breeding. 2014 Jun;34(1):217-30. DOI 10.1007/s11032-014-0031-5

23. Van Ooijen JW. MapQTL® 5, Software for the mapping of quantitative trait loci in experimental populations. Kyazma BV, Wageningen. 2004;63. 
24. Emeriewen O, Malnoy M, Richter K, Kilian A, Hanke MV, Peil A. Evidence of a major QTL for fire blight resistance in the apple wild species Malus fusca. InXIII International Workshop on Fire Blight 10562013 Jul 2 (pp. 289-293). doi: 10.17660/ActaHortic.2014.1056.49

25. Velasco R, Zharkikh A, Affourtit J, Dhingra A, Cestaro A, Kalyanaraman A, Fontana P, Bhatnagar SK, Troggio M, Pruss D, Salvi S. The genome of the domesticated apple (Malus $\times$ domestica Borkh.). Nature genetics. 2010 Oct;42(10):833-9. Chagné D, Orellana-Torrejon C, Saeed M, Troggio M, et al. Mapping of fire blight resistance in Malus $\times$ robusta 5 flowers following artificial inoculation. BMC plant biology. 2019 Dec;19(1):1-2. https://doi.org/10.1186/s12870-019-2154-7 